\title{
REAL-TIME SPECTROSCOPY OF ACOUSTIC WAVES OR MUSIC AS PHYSICAL PHENOMENON
}

\author{
P. ZiELIŃSKI
}

Institute of Nuclear Physics, Radzikowskiego 152, 31-342 Kraków, Poland

The sounds used in music have discrete spectra consisting of a fundamental frequency and of its integer multiples. This is the reason for an affinity of the sounds for which the ratio of the fundamental frequencies is given by a fraction of small integers. Two possible physical mechanisms for this affinity are discussed: (1) an influence of beats between some partial tones and (2) nonlinear interactions between partial tones. Some facts from the music theory are then explained as resulting from the invariance of the most consonant intervals between affine sounds against variations of the timbre and of the register. An analogy is indicated between the classical cadence and the lock-in effect in incommensurate crystals. The lecture is illustrated with extracts from the J.S. Bach Das Wohltemperierte Klavier, the W.A. Mozart sonata for piano KV 570 and a recitative from the Haendel Messiah.

PACS numbers: 43.75. $+\mathrm{a}, 43.66 .+\mathrm{y}, 43.64 .+\mathrm{r}$

\section{Introduction}

The sounds used in the polyphonic and harmonic music are produced by one-dimensional standing waves generated in almost dispersionless objects such as strings and columns of air. The spectrum of every such sound consists of a fundamental frequency and of its integer multiples called higher harmonics or partial tones. The distribution of the intensity among particular harmonics in the spectrum of a given sound determines its timbre. It was established that the dependence of the timbre on the phase shifts of the harmonics is very weak [1]. The pitch of a sound depends on its fundamental frequency in an approximately logarithmic way so that the difference in pitch of two sounds, called musical interval, corresponds to a given ratio of the fundamental frequencies.

One can easily verify, using e.g. a monochord, that the sensation produced by two simultaneous sounds whose fundamental frequencies form a ratio of small integers, such as 2:1, 3:2, 4:3 etc., is pleasant, harmonious and mild, whereas the ratios of the fundamental frequencies expressed by higher integers or by irrational numbers give an unpleasant and rough sensation. In the former case one speaks of consonance and in the latter of dissonance. 


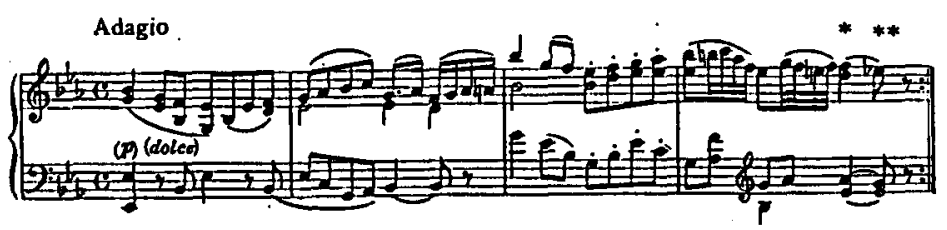

Fig. 1. The beginning of the slow movement of the W.A. Mozart sonata for piano KV 570. The accord $*$ shows a strong tendency to be followed by the accord $* *$.

The rules of the counterpoint and of the harmony require that a dissonance should be solved onto a consonance. An example of that is given in Fig. 1 which is an extract from the Mozart sonata for piano KV 570. Listening to the sequence of accords marked by the asterisks $*$ and $* *$ one can ask whether the self-evident tendency of the first of the accords to be followed by the second one is a result of our habit fixed by tradition or it stems from some deeper natural laws.

\section{Helmholtz's model for the perception consonance}

The first attempt to answer this question on the basis of a physical model has been given by von Helmholtz in his famous book on the sound sensations [2].

The model of Helmholtz summarizes his observations of the anatomy of the ear. The organ responsible for the pitch perception is located in the cochlea. The latter is a tube wound in a spiral form and filled with a liquid. The tube is divided into two parts by a membrane, called basilar membrane, parallel to the axis of the tube. The basilar membrane consists of transverse fibers, each of them attached to an ending of an auditory nerve.

The assumption of Helmholtz was that every fibre of the basilar membrane operates as a resonator "tuned" on one particular frequency in analogy to the row of strings in a harp. Whenever a sound of a given frequency is sent to the ear, the corresponding fibre starts to vibrate as a result of the resonance. Since the membrane is immersed in a liquid, every such resonance has a certain width.

Now if the membrane is stimulated at the same time, by two different frequencies, two resonances arise. The sensation of roughness or dissonance is related, according to Helmholtz, to the beats provoked at those locations of the basilar membrane which are excited simultaneously by two different frequencies. This happens whenever the resonance curves corresponding to different frequencies overlap. If the frequencies of the incident sounds are well separated, the frequency of beats is too high to be perceived because of the limited time resolution of the human senses. Moreover, the amplitude of beats then is relatively small. In the other limit case of both frequencies very close to each other the frequency of beats, being the difference of the incident frequencies, is small and is perceived as a pleasant waving of the sound - a "tremolo". However, when the difference of the frequencies approaches about $33 \mathrm{~Hz}$, the sensation becomes rough analogously to the sensation produced by the consonant " $\mathrm{r}$ " pronounced in Polish, Spanish or Italian manner.

Helmholtz proposed an analytical expression for the sensation of the roughness as a function of the difference in frequency. The corresponding curve is shown 
in Fig. 2. The region in which the curve differs significantly from zero has been called "critical band". As it has been shown by Plomp [3] the width of the critical band depends somewhat on the average of the incident frequencies.

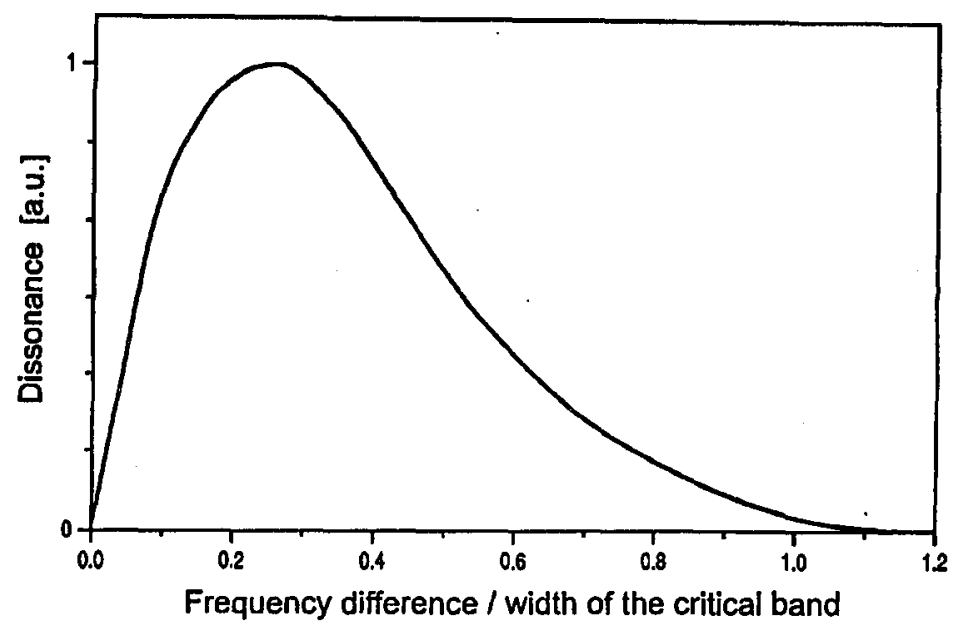

Fig. 2. Dissonance (roughness) of two simple tones as a function of the difference of their frequencies normalized to the width of the critical band (see Refs. [3] and [5]).

In order to get the curve representing the roughness of two complex sounds one has to add the roughness produced by all pairs of the partial tones involved. The resulting curve depends on the timbre of the sounds in such a way that the roughness increases whenever two strong partial tones are separated by a frequency difference close to the maximum of the curve from Fig. 2. An example of the roughness curve for two sounds in which the intensity of the partial tones are inversely proportional to their frequencies is given in Fig. 3. The fundamental frequency of the lower sound is constant and equals to $250 \mathrm{~Hz}$ whereas the fundamental frequency of the higher sound increases continuously from $250 \mathrm{~Hz}$ to 500 $\mathrm{Hz}$. One can immediately remark the minima corresponding to simple fractions of the fundamental frequencies.

The model of Helmholtz involves some assumptions which could not have been verified with the technical means available in his times. Newer studies by von Bekesy [4] have in principle confirmed the hypothesis of the local excitation of the basilar membrane for a given frequency. However, the excited regions determined by von Bekesy are larger than the resonances of the width phenomenologically estimated by von Helmholtz. To explain this discrepancy some studies have been done on the reaction of nerves to the strength of stimuli. It turned out that the reaction is nonlinear so as the stronger stimuli are felt unproportionally stronger with respect to the weak stimuli. Consequently, the final sensation is much more localized than the regions of excitation of the basilar membrane. This finding can be in favour of the Helmholtz theory, which with the newer modifications is known as theory of the localization of the pitch sensation [5]. 


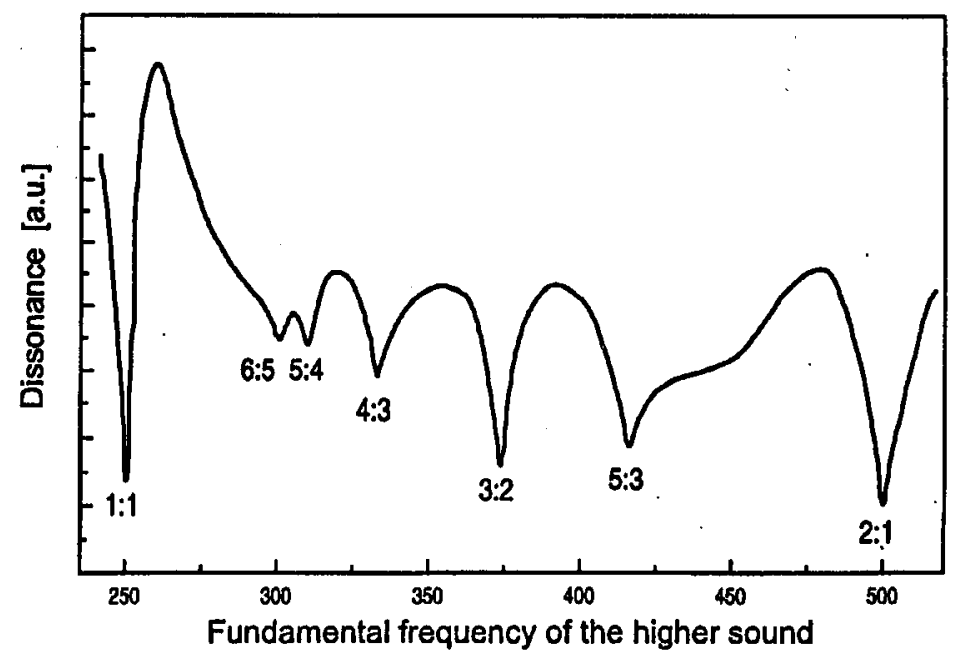

Fig. 3. Dissonance of two complex sounds as a function of the difference of their fundamental frequencies. The curve obtained from the data given in Ref. [5].

In fact there exist some alternative theories on this subject. The present studies are focussed on the motions of the liquid (perylymfe) filling the cochlea. These are apparently nonlinear waves mostly influenced by the end orifice between two parts of the cochlea - helicotrema [6].

\section{Nonlinear interactions of partial tones}

The analysis of the beats is not the unique way of obtaining a curve showing a character represented in Fig. 3, i.e. marked by minima at the ratios of small integers. Another idea is to take into account the interactions of the particular harmonics. This is known from the theory of the incommensurate crystals, where the modulation wave interacts with the underlying crystal lattice through so-called umklapp terms, which become particularly important for the ratios of the wavelengths expressed by small integers [7]. The phases described by such simple fractions are particularly stable. Whereas the incommensurate modulation varies continuously with temperature, the commensurate, so-called lock-in phases, subsist within some definite regions of temperature. By this does the nature satisfy the rule of harmony requiring that a dissonance should be solved onto a consonance. The lock-in phases thus correspond to cadences in the classical music.

In order to built a theory based on the principle of the umklapp terms one has to determine the form of the interactions of the partial tones. The physical reason for such an interaction lies in the known nonlinearity of the ear. The simplest idea is to assume that the interaction is proportional to the product of the amplitudes of the partial tones independently of their frequencies. This quantity has been called concordance and calculated by Chouvel [8]. Such a theory requires an assumption that the partial tones should be represented by Gaussian peaks around their frequencies, otherwise, e.g. a slightly mistuned unison would be a strong dissonance, whereas in reality it gives an impression of a single sound slightly waving 
because of the beats as it is predicted by the theory of Helmholtz. The meaning of the widths of the peaks and their relation to other physical properties are not known for the moment.

In any case, both linear phenomenon of beats and the nonlinear interactions of the partial tones seem to contribute to the sensation of dissonance and consonance. The simplest evidence for that is that a dissonance and consonance are also distinguishable in the music played by flutes, which are known to produce sounds composed practically of the fundamental tones only. The higher harmonics essential as well in the beats theory as in the nonlinear interaction theory must, therefore, be created in the ear.

\section{Affinity of sounds}

As we have seen various theories have been proposed to explain the consonant character of sounds whose fundamental frequencies are in a ratio described by small integers. The sounds separated by the most consonant intervals show an affinity which is incontestable whatever might be the physical origin of the curve from Fig. 3.

\subsection{Octave - the absolute consonance}

The most pronounced affinity corresponds to the ratio $2: 1$ of the fundamental frequencies i.e. to the interval of octave. The spectra of two sounds separated by an octave is shown in Fig. 4. The highest degree of affinity of the sounds separated by an octave is easily understandable within the theory of beats. Indeed, the smallest difference in frequencies in this case then is equal to $f_{0}$. Therefore the

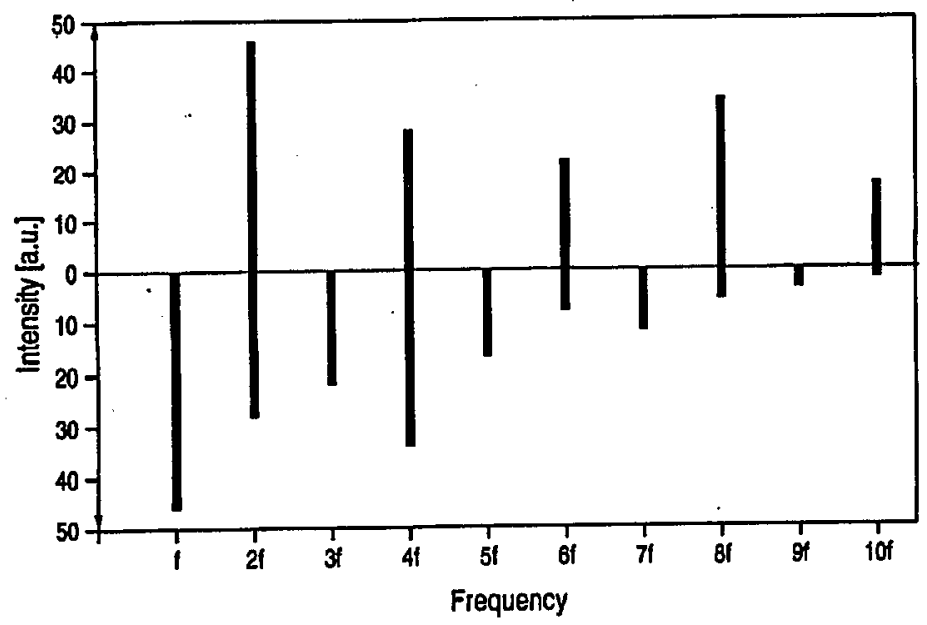

Fig. 4. The spectra of sounds of the same timbre and separated by the interval of one octave. The fundamental frequency of the higher sound $2 f$ coincides with the second partial tone of the lower sound $f$. 
frequency of beats is equal to the fundamental frequency of the lower sound. Such beats do not produce any waving of the intensity but only modify the amplitude of the fundamental frequency. Also nonlinear interactions between the harmonics produce some combination tones which are separated by multiples of the octave with respect to the incident harmonics. This absence of beats is independent on the sound timbre as well as on the register. In physical terms, one can say that the consonance of octave is invariant against variations of the timbre and of the register. The latter invariance can be called scaling symmetry.

There is an interesting analogy between the consequences of the invariance in physics and in the music theory. In physics, any invariance is related to a conservation law. This is the case in the discussed invariance. The conserved quantity is the extent of the musical scales in all very distant and isolated cultures: all the musical scales are contained within one octave. In the European system the sounds separated by multiple of octaves bear the same names: do, re, mi, fa etc. The equivalence of the sounds separated by octaves can be noticed in the simplest way by observing how amatour singers displace the tune whenever the sung melody exceeds the scale of their voice: they simply displace the tune by an octave. A specific interplay between the timbre and pitch has allowed for creation of so called acoustic paradoxes. A sequence of sounds of a very special timbre may, e.g., give an impression of a continuous ascent although it consists of a finite number of sounds [9].

\subsection{Fifth - a perfect consonance}

The second-deepest minimum in the curve from Fig. 3 occurs at the frequency ratio $3: 2$ and corresponds to the musical interval of fifth. The history of the European music is a series of consequences of this affinity of sounds. The first polyphonic music - organum was based on the parallel fifths. The ending accords of the medieval and renaissance pieces were built of octaves and fifths. The most important musical forms are based on this interval: indeed the subject of a fugue - the $d u x$ is repeated in the interval of the fifth - the comes. Similarly the second theme of the classical sonata is in the tonality of the dominant, so that displaced by a fifth with respect to the first theme. Finally, the ubiquitous perfect cadence in the classical music is a sequence of accords based on the sounds separated by a fifth. The jump of a fifth has an analogy to the steps in surface physics. From among all the conceivable steps the one by fifth has the smallest energy and, therefore, the highest probability to occur.

\subsection{Thirds and sixths - imperfect consonances}

The thirds and sixths are nice consonances for some timbres but may become rough for some other timbres, especially for organ mixtures having particularly strong higher harmonics. Also a shift of these intervals towards deeper registers may reduce their consonant character, because the beats among some harmonics then fall into the critical band. Consequently, one cannot speak any more of an invariance against changes of timbre and of the register for these intervals. As a result, the use of these intervals varies for different cultures. In the European 
classical music the thirds and sixths soften the contrast between the dissonant and consonant accords.

\subsection{Dissonances}

According to the rules of the counterpoint and harmony, the dissonant intervals: the seconds, sevenths and the triton convey a tension which should be released by a solution onto a consonance. In this respect the rules of harmony "predicted" in a way the mentioned behaviour of the incommensurate phases which relax to the lock-in structures. The use of the dissonances is characteristic of the music of the twentieth century. The composers try to expose the audience to sounds which show a lower affinity to attain an expression different from what has been already exploited in the classical rules based on consonances. This does not contradict the existence of the sounds affinity, but shows that their application may differ in different styles of music.

\section{Concluding remarks}

We have given an example of an analogy between rules of harmony: a necessary solution of dissonances onto consonances, and the lock-in phenomenon in the incommensurate crystals. In a way music has "discovered" this effect much earlier than the crystallographers. An important difference is that in the music the expression is a sequence of accords, whereas in physics it has a verbal expression assisted by mathematical formulas.

In the same spirit, one can think of the quantum mechanics as of a rebirth of the idea of the universal harmony. In fact the nature of microparticles such as electrons or nuclei have more to do with the waves, so essential in music, than with the corpuscular concepts characteristic of the classical mechanics.

The beauty of music and of physics has something in common. This is the charm of a regularity which sometimes emerges from a rich variety of apparently incoherent phenomena.

\section{References}

[1] J.R. Pierce, E.E. David, Świat dźwięków, PWN, Warszawa 1967 (in Polish).

[2] H.L.F. von Helmholtz, Die Lehre von den Tonempfindungen als physiologische Grundlage der Theorie der Musik, F. Vieweg und Sohn, Braunschweig 1895.

[3] R. Plomp, J. Acoust. Soc. Am. 36, 1628 (1964); R. Plomp, W.J.M. Levelt, J. Acoust. Soc. Am. 38, 548 (1965).

[4] G. von Bekesy, Experiments on Hearing, McGraw-Hill, New York 1960.

[5] J.R. Pierce, The Science of Musical Sound, Scientific American Library, W.H. Freeman, New York 1983.

[6] F. Schick, Acoustica 80, 463 (1994).

[7] K. Parliński, K.H. Michel, Phys. Rev. B 29, 396 (1984).

[8] J.M. Chouvel, to be published, 1995.

[9] R.N. Shepard, Psychological Rev. 89, 305 (1982); D. Deutsch, Music Perception 8, 335 (1991). 